\title{
Targeted disruption of S100P suppresses tumor cell growth by down-regulation of cyclin D1 and CDK2 in human hepatocellular carcinoma
}

\author{
JEONG KYU KIM ${ }^{1}$, KWANG HWA JUNG ${ }^{1}$, JI HEON NOH ${ }^{1}$, JUNG WOO EUN ${ }^{1}$, HYUN JIN BAE ${ }^{1}$, HONG JIAN XIE ${ }^{1}$, \\ YOUNG MIN AHN ${ }^{2}$, JAE CHUN RYU ${ }^{3}$, WON SANG PARK ${ }^{1}$, JUNG YOUNG LEE ${ }^{1}$ and SUK WOO NAM ${ }^{1}$ \\ ${ }^{1}$ Department of Pathology, Microdissection Genomics Research Center, College of Medicine, The Catholic \\ University of Korea; ${ }^{2}$ Department of Kidney System, College of Oriental Medicine, Kyung Hee University; \\ ${ }^{3}$ Cellular and Molecular Toxicology Lab, Korea Institute of Science and Technology, Seoul, Korea
}

Received June 26, 2009; Accepted August 24, 2009

DOI: 10.3892/ijo_00000442

\begin{abstract}
Hepatocellular carcinoma (HCC) is the third leading cause of cancer death worldwide. The number of cases of $\mathrm{HCC}$ has continued to increase in recent decades. Previous studies have suggested that S100P, a member of the S100P calcium-binding protein family, is aberrantly regulated in several malignant neoplasms. However, the underlying molecular mechanisms of the dysregulation of S100P remain to be elucidated. To investigate biological effects of S100P on hepatocarcinogenesis, aberrant expression of S100P was investigated by immunohistochemistry (IHC), Western blot analysis and reverse transcriptase-polymerase chain reaction (RT-PCR) in HCC tissues and cell lines. Endogenous expression of S100P was disrupted by the RNA interferencemediated protein knockdown method in the human Hep3B liver cancer cell line. Then, cell growth and cellular apoptosis were compared with control siRNA transfectants. The effects of S100P-silencing on the major components of cell cycle regulation were assessed by Western blot analysis. As results, elevated levels of S100P were observed in the HCC tissues compared to the corresponding normal tissues. Targeted disruption of S100P suppressed cell growth and augmented cellular apoptosis. In addition, inhibition of S100P resulted in the down-regulation of cyclinD1 and CDK2. In conclusion, this study showed over-expression of S100P in HCC. The aberrant regulation of S100P in HCC might activate cyclin D1 and CDK expression and contribute to the mitogenic
\end{abstract}

Correspondence to: Dr Suk Woo Nam, Department of Pathology, College of Medicine and Microdissection Genomics Research Center, The Catholic University of Korea, 505 Banpo-dong, Seocho-gu, Seoul 137-701, Korea

E-mail: swnam@catholic.ac.kr

Key words: S100P, hepatocellular carcinoma, apoptosis, cell growth, cell cycle potential of tumor cells during HCC carcinogenesis. These findings provide information that suggests new therapeutic strategies for the treatment of liver cancer.

\section{Introduction}

Human hepatocellular carcinoma (HCC) is one of the most common neoplasms. HCC is currently the third leading cause of cancer death worldwide (1). Most patients with HCC have a poor prognosis and die within several months after the diagnosis (2,3). Recent studies have found that genetic alterations of tumor suppressor genes or oncogenes such as p53, $\beta$-catenin and AXIN are involved in hepatocarcinogenesis (4-6); however, the frequency of mutations, of these genes, appears to be very low in patients with HCC. Furthermore, it is unclear how these genetic changes correspond to the clinical characteristics of individual patients with HCC. Therefore, the mechanisms underlying the carcinogenesis of $\mathrm{HCC}$ remain unclear.

The S100 proteins belong to the EF-hand superfamily of $\mathrm{Ca}^{2+}$-binding proteins and include: S100A2, S100A4, S100A6, S100A7, S100B and S100P. These proteins are mediated by $\mathrm{Ca}^{2+}$-dependent signal transduction pathways involved in the regulation of the cell cycle, growth, differentiation and metabolism (7-10). The S100 proteins have been functionally associated with a variety of neurological, cardiac and neoplastic diseases. The S100 calcium-binding protein P (S100P), a 95-amino acid protein, was first purified from the placenta with limited cell distribution in other tissues $(11,12)$; it is one of the latest members of the S100 family identified and consists of $\mathrm{Ca}^{2+}$-binding proteins of the EF-hand type $(9,13)$. The functions of the members of this family of proteins vary widely. The primary function of S100P is to mediate the $\mathrm{Ca}^{2+}$-dependent signal transduction cascades involved in cell growth and differentiation, cell cycle regulation and metabolic control $(8,10)$. Many studies have shown that S100P plays a role in carcinogenesis (14-16), and its expression has been shown to increase in several malignancies including those of the colon (17), breast (18), pancreas (19), prostate (20) and lung (21). In pancreatic cancer, S100P is over-expressed due 
to hypomethylation of specific genes (22). In prostate cancer, $\mathrm{S} 100 \mathrm{P}$ expression is regulated by androgens (20) and interleukin-6 (23). In gastric cancer cell lines, S100P expression has been shown to be induced by retinoic acid (24). In breast cancer cell lines, S100P levels have been associated with immortalization of cells (18). In colon cancer cell lines, S100P expression has been correlated with resistance to chemotherapy (25). In lung cancer, S100P expression has been correlated with decreased patient survival (26). It has been shown that S100P can induce anchorage-independent tumor cell growth in vitro and improve tumor growth in a xenograft model. These results suggested that S100P functionally participates in the control of tumorigenic potential in vivo (15). However, despite these observations, it is not clear what the specific molecular events are that underly the aberrant regulation of S100P in most cancers, including HCC.

The aim of this study was to investigate the biological role of S100P in HCC development and progression. The expression of S100P in HCC was compared to corresponding normal hepatocytes. In addition, S100P expression was disrupted by siRNA and the regulatory mechanisms of the cell cycle component, in particular the G1/S transition of the Hep3B cells, was evaluated.

\section{Materials and methods}

Tissue samples. Five frozen human HCCs and their corresponding normal tissues were obtained from Korean patients, and samples were randomly selected for this study. Approval was obtained from the institutional review board of The Catholic University of Korea, College of Medicine. Informed consent was provided according to the Declaration of Helsinki.

Cell culture and reagents. Hep3B, SNU182, SNU354, SNU387, SNU449 and SNU475 cells were purchased from Korean Cell Line Bank (KCLB; Seoul, Korea) and THLE-3, HepG2, PLC/PRF/5 and CHANG cells were purchased from the American Type Culture Collection (ATCC; Manassas, VA, USA), respectively. Each cell line was grown in RPMI1640 medium supplemented with $10 \%$ fetal bovine serum (Sigma, St. Louis, MO, USA). Cells were trypsinized, and then seeded at $5 \times 10^{5}$ cells/plate in a $100 \mathrm{~mm}$ dish, and allowed to grow overnight at $37^{\circ} \mathrm{C}$ in a humidified incubator at $5 \%$ $\mathrm{CO}_{2}$. At $24 \mathrm{~h}$ after plating, the cells were transfected with S100P-specific siRNAs in Opti-MEM (Life Technologies, Gaithersburg, MD, USA). Transfection was carried out using oligofectamine reagent (Invitrogen, Carlsbad, CA, USA) according to the manufacturer's specifications.

Reverse transcriptase-polymerase chain reaction (RT-PCR). Total RNA was isolated using TRIzol reagent (Invitrogen) according to the manufacturer's instructions. RNA concentration was determined by NanoDrop (Thermo Fisher Scientific Inc., Waltham, MA, USA). Next, $1 \mu \mathrm{g}$ of total RNA was reverse transcribed by Superscript II enzyme (Gibco-BRL, Gaithersburg, MD, USA) with $0.5 \mu \mathrm{g}$ oligo(dt) (Amersham Biosciences, Piscataway, NJ, USA). The reaction mixture was incubated at $42^{\circ} \mathrm{C}$ for $60 \mathrm{~min}$ and then at $72^{\circ} \mathrm{C}$ for $15 \mathrm{~min}$. To normalize differences in the amount of total cDNA added to each reaction, glyceraldehydes-3-phosphate dehydrogenase (GAPDH) gene expression was used as an endogenous control. The cycling conditions were as follows: initial denaturation at $95^{\circ} \mathrm{C}$ for $10 \mathrm{~min}$, followed by 40 cycles at $95^{\circ} \mathrm{C}$ for $15 \mathrm{sec}, 58^{\circ} \mathrm{C}$ for $5 \mathrm{sec}, 72^{\circ} \mathrm{C}$ for $5 \mathrm{sec}$. S100P and GAPDH transcripts were measured using polyacrylamide gel electrophoresis.

Western blot analysis. The following antibodies were used: anti-S100P (BD Biosciences, San Jose, CA, USA), antiPARP, anti-p15/16/21, anti-cyclinD1/D3 (Cell Signaling Technology, Danvers, MA, USA), anti- $\alpha$-tubulin (Sigma), anti-CDK2 (Santa Cruz Biotechnology, Santa Cruz, CA, USA), anti-mouse IgG (Amersham Biosciences), anti-rabbit IgG (Bio-Rad Laboratories, Hercules, CA, USA) and ECL plus Western blotting detection system (Amersham Biosciences) were used to detect the bound antibodies. The intensity of the Western blot bands was quantified using LAS 3000 (Fuji Photo Film Co., Tokyo, Japan).

Immunohistochemistry of the S100P. For the immunohistochemical analysis, we used HCC tissue micro array (TMA) slides. The S100P primary antibody (1:25 dilution) was used in the IHC. Diaminobenzidine (DAB; Dako, Copenhagen, Denmark) was used as a chromogen, and the slides were then lightly counterstained with Mayer's hematoxylin. Scoring of the TMA was performed independently by two pathologists. In the event of disagreement, the two reached a consensus by jointly re-evaluating the TMA using a multi-head microscope. The intensity of the immunostaining was graded as: negative, weak positive, $1+$ (moderate), $2+$ and $3+$ (strong). If the number of immunostained cells was less than $10 \%$, the sample was considered negative.

S100 calcium binding protein $P(S 100 P)$ gene silencing by small interfering RNA. S100P-specific small-interfering RNAs (siRNA) were designed and purchased through Silencer ${ }^{\circledR}$ Pre-designed siRNAs (Ambion, Austin, TX, USA) to transfect Hep3B cells with a siRNA targeted against the S100P gene (S100P siRNA sense sequence: GCCGUGGAU AAAUUGCUCA dTdT, antisense sequence: UGAGCAAUU UAUCCACGGC dAdT and negative control siRNA sense sequence: GAUAUAGGAAGUCCAUACU dTdT, antisense sequence: AGUAUGGACUUCCUAUAUC dTdT).

MTS assay for cell proliferation. Proliferation of cells was measured at indicated time with the use of Cell Titer96 Aqueous One solution cell proliferation assay (MTS; Promega, Madison, WI, USA), according to the manufacturer's instructions. Briefly, Hep3B cells were seeded in 12-well flat-bottomed microtiter plates at a density of $2 \times 10^{4}$ cells/ well. The cells were transfected with siRNA targeting S100P or scramble siRNA, and then cells were allowed to grow for indicated time points and were then incubated with MTS for $1 \mathrm{~h}$ at $37^{\circ} \mathrm{C}$ in a humidified $5 \% \mathrm{CO}_{2}$ atmosphere. The absorbance was read at $490 \mathrm{~nm}$ by VICTOR $3^{\mathrm{TM}}$ Multilabel Plate Readers (Perkin-Elmer, Waltham, MA, USA). All measurements were performed in triplicate and each experiment was repeated at least three times. 
A
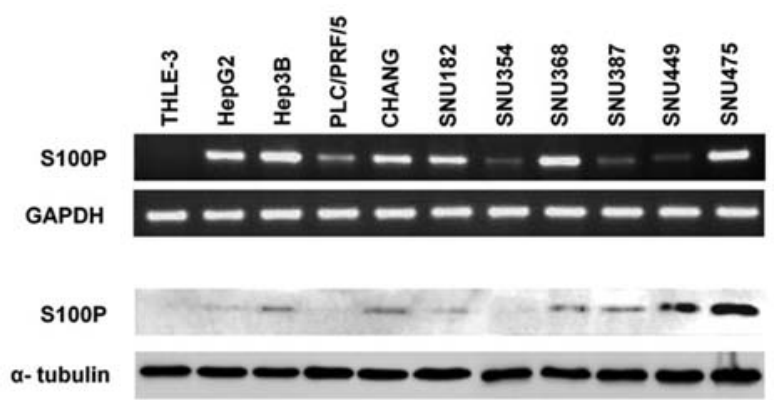

B

\begin{tabular}{|c|c|c|c|c|c|c|c|c|c|c|}
\hline \multirow[t]{2}{*}{ Sample No. : } & \multicolumn{2}{|c|}{152} & \multicolumn{2}{|c|}{209} & \multicolumn{2}{|c|}{272} & \multicolumn{2}{|c|}{384} & \multicolumn{2}{|c|}{436} \\
\hline & $\mathbf{N}$ & $T$ & $\mathrm{~N}$ & $T$ & $\mathrm{~N}$ & $T$ & $\mathrm{~N}$ & $T$ & $\mathbf{N}$ & $T$ \\
\hline S100P & 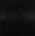 & $n$ & 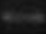 & 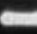 & n & & 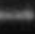 & 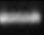 & & $m$ \\
\hline
\end{tabular}

Figure 1. Expression of S100P in hepatocellular carcinoma tissues and cell lines. Human liver tissues from five patients with HCC (patients are shown by number and identified by one letter, $\mathrm{N}$, corresponding normal tissue, $\mathrm{T}$, tumor tissue), 10 liver cancer-derived cell lines and THLE-3 immortalized cell lines from human hepatocyte were collected and harvested for isolation of total proteins or RNAs. (A), Reverse transcription-polymerase chain reaction (RT-PCR) showing the expression of S100P mRNA in hepatocytes and HCC cell lines. GAPDH served as an internal control (upper), and Western blot analysis shows the expression of S100P protein in hepatocytes and liver cancer cell lines. For the Western blot analysis, $\alpha$ tubulin served as an internal control (bottom). Data are representative of at least two independent experiments. (B), RT-PCR and Western blot analysis showing the expression of S100P mRNA and protein in five selected HCC tissues. Data are representative of at least two independent experiments.

Cell cycle and apoptosis analysis. After transfection, the Hep3B cells $\left(2 \times 10^{5}\right)$ were harvested by trypsinization at $48 \mathrm{~h}$ after transfection, and then washed with $1 \mathrm{X}$ PBS, followed by fixed in $70 \%$ alcohol for 1 day at $-20^{\circ} \mathrm{C}$. After fixation, the cells were washed with ice cold $1 \mathrm{X}$ PBS twice and incubated for $30 \mathrm{~min}$ in PBS containing RNase A $(10 \mathrm{mg} / \mathrm{ml})$ at $37^{\circ} \mathrm{C}$. After the RNase A treatment, the nuclei were stained with $5 \mathrm{mg} / \mathrm{ml}$ of propidium iodide (PI) before their DNA content was measured using a flow cytometer (BD Biosciences). The Annexin V-FITC Apoptosis Detection Kit I (BD Biosciences) was used to quantify the level of apoptosis in the samples. Briefly, the cells were trypsinized, washed twice with cold PBS and resuspended in $1 \mathrm{X}$ binding buffer at a concentration of $1 \times 10^{6}$ cells $/ \mathrm{ml}$, and $100 \mu 1$ of the solution $\left(1 \times 10^{5}\right.$ cells $)$ was transferred to a $5 \mathrm{ml}$ culture tube and $5 \mu \mathrm{l}$ of Annexin V-FITC solution was added; PI $(10 \mu \mathrm{l})$ was added to each tube and the cells were analyzed by flow cytometry.

Statistical analysis. Each experiment was performed at least three times. The data are presented as the mean \pm standard errors for the number of experiments. Statistical significance was defined by the results of the unpaired Student's t-test at a $\mathrm{p}<0.05$ and the Chi-square test for the immunohistochemistry.

\section{Results}

S100P expression in hepatocellular carcinoma. Accumulating evidence has shown that $\mathrm{S} 100 \mathrm{P}$ is functionally associated
Table I. Results of immunohistochemical staining of S100P in HCC.

\begin{tabular}{lcc}
\hline p-value & Normal $(\mathrm{n}=25)$ & $\begin{array}{r}\mathrm{HCC}(\mathrm{n}=25) \\
\mathrm{p}=0.0209^{\mathrm{a}}\end{array}$ \\
\hline S100P, n (\%) & & \\
S100P negative & $14(56)$ & $6(24)$ \\
S100P positive & $11(44)$ & $19(76)$ \\
\hline
\end{tabular}

${ }^{\mathrm{a} C h i-s q u a r e ~ t e s t . ~}$

with a variety of neoplasms; however, its role in human hepatocellular carcinoma has not yet been determined. Thus, in order to investigate aberrant expression of S100P in HCC, we measured and compared the expression levels of S100P in both liver cancer cell lines and human HCC tissues. In the liver cancer cell lines, when we assessed the expression of S100P by RT-PCR and Western blot analysis, most of the cancer cell lines derived from HCC showed a higher expression of S100P when compared to THLE-3 cells that were immortalized from normal hepatocytes and used as normal control cells for comparison. In the normal cells, S100P was not detectable by either RT-PCR or Western blot analysis. In addition, for the HepG2 cells, established from hepatoblastoma, there was a high expression of S100P (Fig. 1A). In order to confirm the aberrant expression of S100P in the HCCs, the differential expression of S100P was determined in five selective human HCC tissues. As shown in Fig. 1B, $\mathrm{S} 100 \mathrm{P}$ was observed to be over-expressed in the tumor tissues compared to the corresponding normal tissues.

The protein expression of S100P was further validated by immunostaining the HCC specimens with S100P antibody; the results are summarized in Fig. 2 and Table I. As summarized in Table I, 19 out of the 25 HCCs tested (76\%) resulted in moderate or strong positive staining with the S100P antibody, while 14 out of the $25(56 \%)$ normal hepatocyes showed negative or weak expression of S100P in the adjacent normal tissues. The higher rate of positive (44\%) staining in the normal tissues could be attributed to background disease affecting the normal tissues such as chronic hepatitis or liver cirrhosis. Fig. 2 shows a representative image of normal hepatocytes (Fig. 2A and C) and HCC tissues (Fig. 2B and D). These results suggest that $\mathrm{S} 100 \mathrm{P}$ is dysregulated in HCCs and may contribute to HCC formation or progression.

Targeted disruption of S100P suppresses neoplastic activity of Hep3B cells. To explain the biological consequence of dysregulation of S100P expression, we targeted S100P by the RNA interference-mediated protein knockdown method, in Hep3B human HCC cells. S100P gene silencing was evaluated by siRNA targeting of S100P, and the efficiency of the S100P-targeted siRNA-mediated down-regulation of S100P mRNA was assessed by RT-PCR. The results showed that both 50 and $100 \mathrm{nM}$ of the S100P siRNA could effectively knock down the expression of S100P in the Hep3B cells compared to the non-silencing (scrambled) 

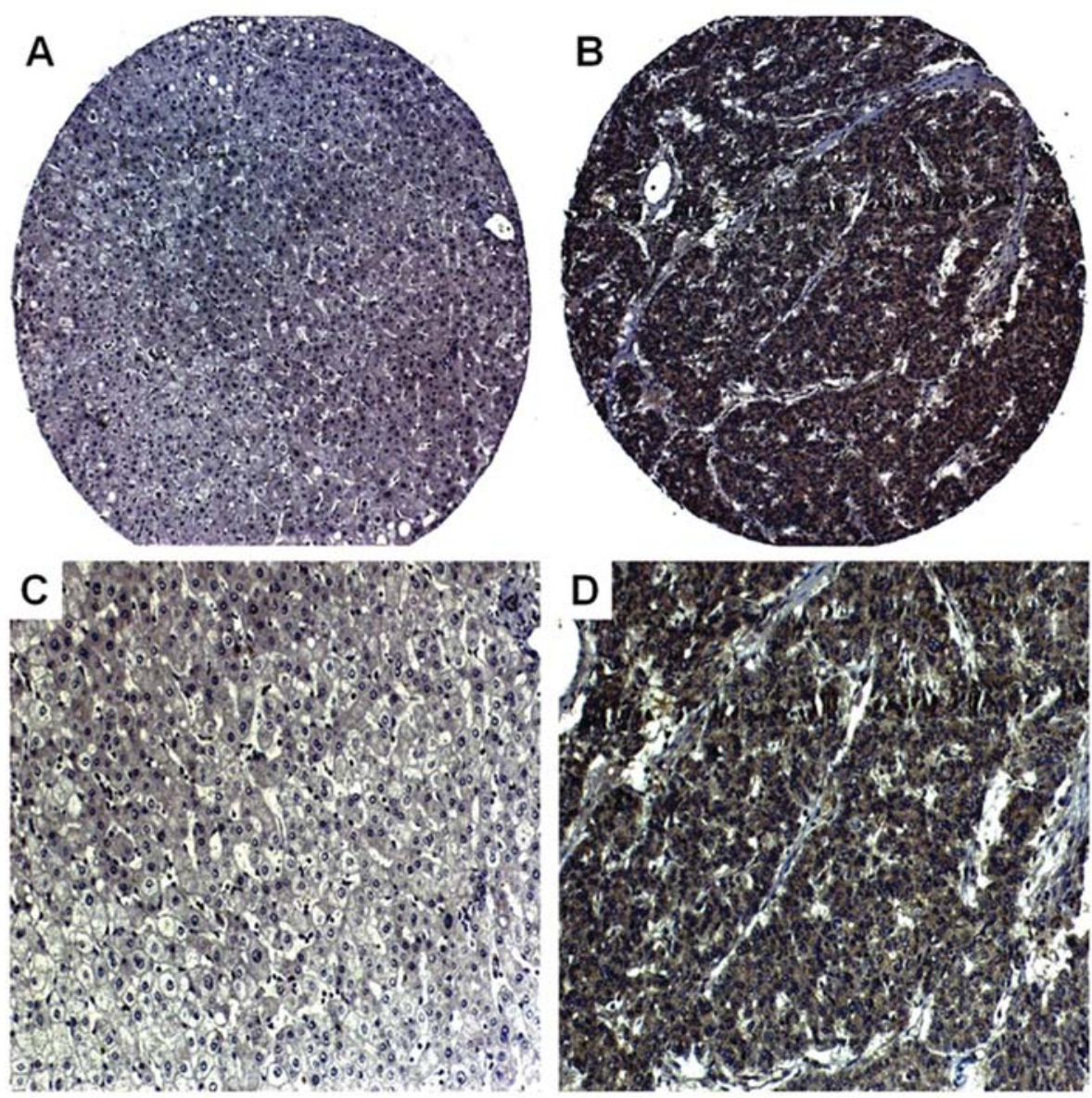

Figure 2. Representative image of immunohistochemistry for S100P in human HCCs and adjacent normal tissues. Immunostaining of HCC and adjacent normal tissues was carried with S100P antibody. The representative images of normal hepatocytes with magnification (A), x100 and (C), x200, and HCC with magnification (B), x100 and (D), x200 were developed with DAB chromogen and counterstained with hematoxylin.

A

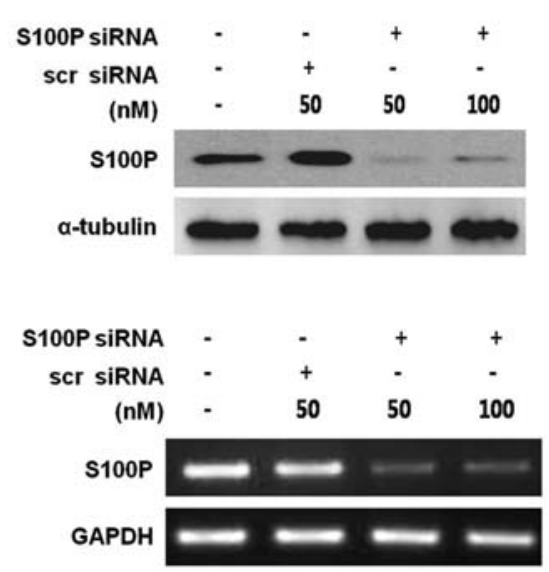

B

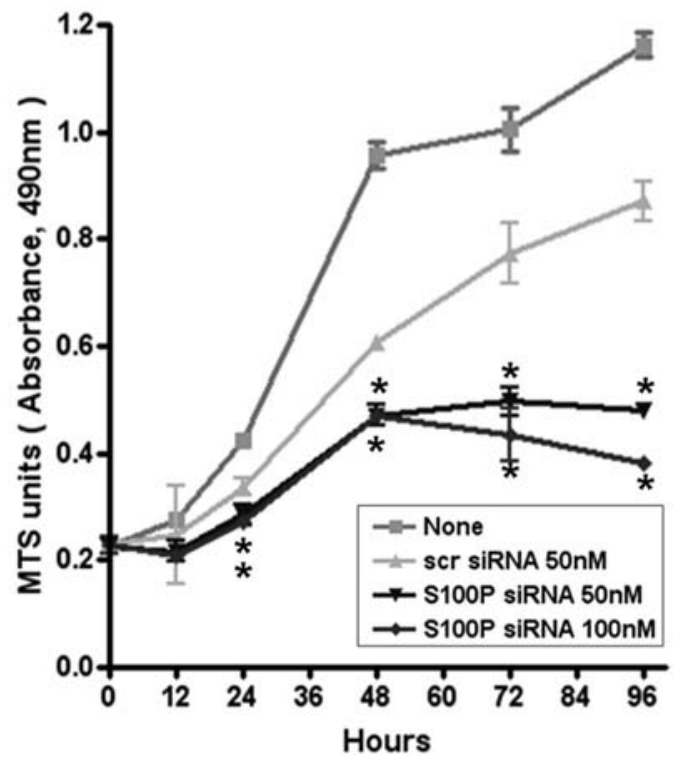

Figure 3. Targeted disruption of endogenous S100P suppresses the growth rate of Hep3B cells. (A), RT-PCR and Western blot analysis showing the silencing of endogenous S100P mRNA and protein levels in Hep3B cells after transfection with the indicated concentration of S100P specific siRNA (S100P siRNA), but not with scrambled siRNA (scr) or no treatment (none). (B), Cell growth rates of Hep3B cells transiently transfected with scrambled siRNA (scr) and S100P specific siRNA (S100P siRNA). The cells were seeded on 24-well plates at an equal density and cultured for the indicated time before the number of each cell type was evaluated by MTS assay. The data are presented as mean \pm standard error for three experiments (unpaired Student's t-test, " $\mathrm{p}<0.05$ vs. control). 


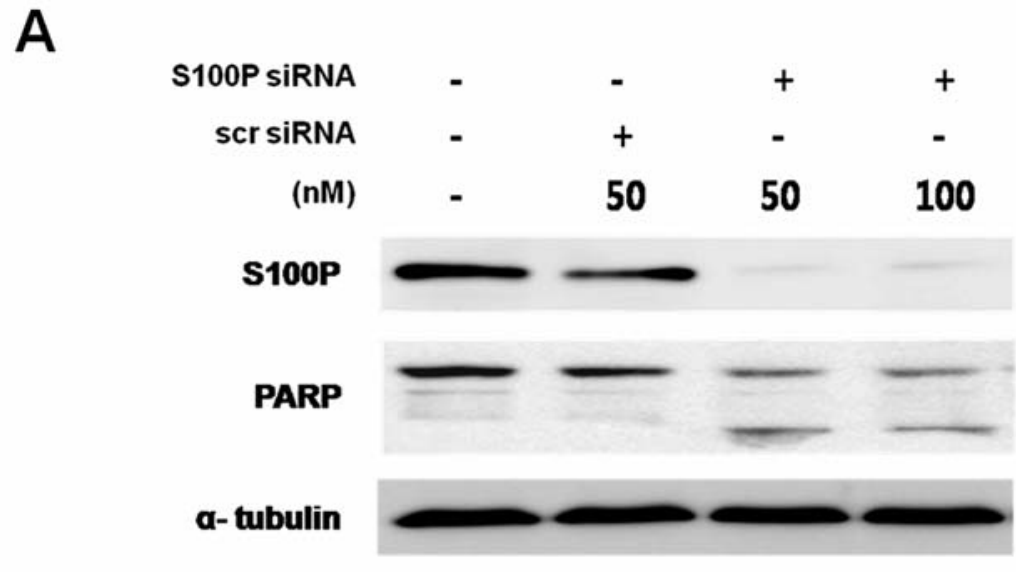

B

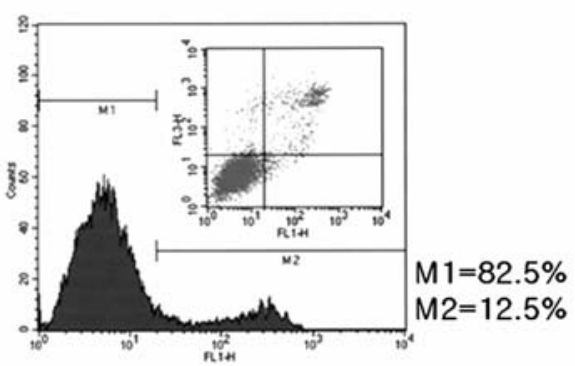

None

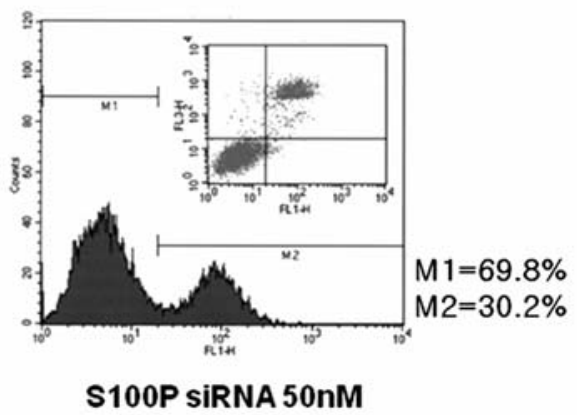

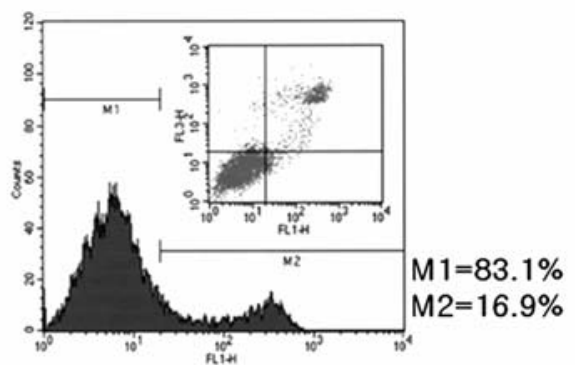

Src SIRNA 50nM

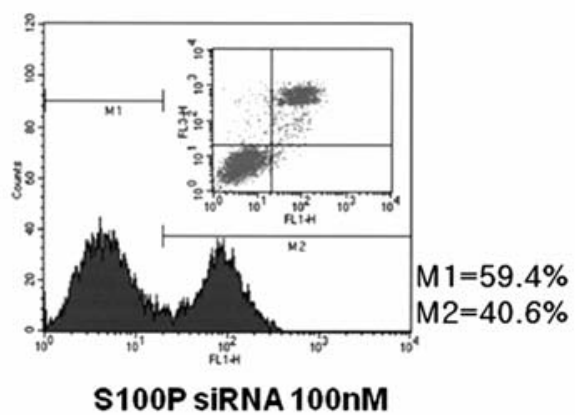

Figure 4. Increase in the basal level of apoptosis in the Hep3B cells after silencing endogenous S100P. (A), Endogenous S100P, in the Hep3B cells, was knocked down by S100P specific siRNA (S100P siRNA). The cleavage form of PARP, a hallmark of apoptosis, was assessed by Western blotting with antibody for PARP. For normalization of protein expression, $\alpha$-tubulin was used as an internal control. (B), Silencing of endogenous S100P increased the basal apoptosis in the Hep3B cells. The Hep3B cells were transiently transfected with scrambled siRNA (scr) or S100P specific siRNA (S100P-siRNA) and stained with Annexin V and propidium iodide and then quantified by fluorescence-activated cell sorter (FACS) analysis. The population shown in the figure is the total distribution of cells. Significant induction of basal apoptosis was observed in the Hep3B cells after silencing of endogenous S100P at both concentrations. Data were obtained in three independent experiments.

siRNA. This observation was confirmed at the protein level by Western blot analysis, which showed that S100P-targeted siRNA could silence protein expression of S100P compared to a negative control siRNA (Fig. 3A). To determine the biological effect of the disruption of S100P expression, the growth rate of the Hep3B cells was determined by MTS assay. As shown in Fig. 3B, silencing of S100P significantly decreased the cell growth rate at both the 50 and $100 \mathrm{nM}$ siRNA concentrations compared to cells treated with the control siRNA. This anti-mitogenic effect on Hep3 cells could be partially explained by disturbance of the cell cycle regulation or augmentation of cellular senescence and apoptosis. In addition, a similar result was observed in the cell viability assay (data not shown).
Next, we examined the effect of S100P, in the Hep3B cells, on cellular apoptosis. As shown in Fig. 4A, when the cells were almost completely knocked down, the cells displayed apoptosis by increasing the cleavage form of poly ADPribose polymerase (PARP), a hallmark of apoptosis. Similar results were obtained by flow cytometric anaysis, measuring the Annexin V staining of cells of the siRNA transfectants. The flow cytometric analysis with propidium iodide (PI) and Annexin V staining, for the dead cells or apoptotic cells, indicated that both early and late stages of apoptosis (M2) were enhanced in the S100P-siRNA transfectant cells. As shown in Fig. 4B, a significant induction of apoptotic cells was seen in the S100P-siRNA transfectants compared to the control groups (none or scr siRNA treatment). The apoptotic 


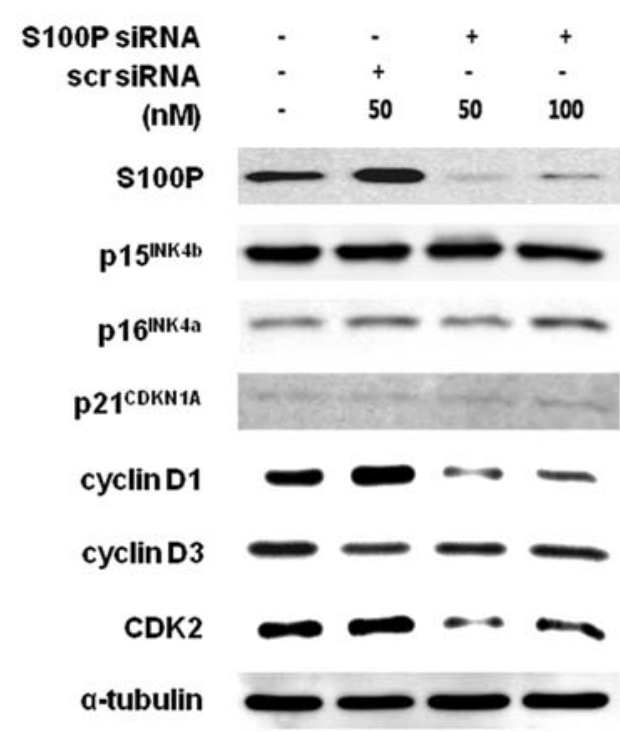

Figure 5. Regulatory mechanisms of S100P associated with the G1/S cell cycle transition. The effect of silencing endogenous S100P on cell cycle regulation. After transfection with the indicated concentration of siRNA targeting S100P (S100P siRNA) or scrambled siRNA (scr), the expression level of p21, p16, p15, cyclin D1, cyclin D3 and CDK2 were assessed by Western blot analysis. Expression of CDK2 and cyclin D1 proteins was decreased after knockdown of endogenous S100P. For normalization of protein expression, $\alpha$-tubulin was used as an internal control. Data are representative of two independent experiments.

cells (right in dot-plot graphs of Fig. 4B) were increased from 30.2 to $40.6 \%$ in the 50 and $100 \mathrm{nM} \mathrm{S100P-siRNA}$ treatment groups compared to no treatment $(12.5 \%)$ or scr siRNA (16.9\%) treatment.

Regulatory mechanisms of S100P on G1/S cell cycle transition. The fact that the suppression of S100P elicits regression of Hep3B cell growth implies that S100P is involved in the regulation of the cell cycle circuit. In addition, FACS analysis with PI staining of cells showed a decrease in the proportion of cells in the S-phase in the S100P-siRNA transfectants (data not shown). Because cyclin-dependent kinases (CDKs), CDK inhibitors (CDKIs) and cyclins are the core components of the cell cycle clock, we next examined the effects of S100P suppression on these regulatory components in the G1/S phase of the cell cycle. In the G1/S phase transition of the cell cycle, it has been well-established that negative cell cycle regulators such as $\mathrm{p} 21^{\mathrm{WAF} / \mathrm{CIP} 1}, \mathrm{p} 15^{\mathrm{INK} 4 \mathrm{~B}}$, $\mathrm{p} 16^{\mathrm{INK} 4 \mathrm{~A}}$ and $\mathrm{p} 27^{\mathrm{Kip} 1}$ are the key modulators that suppress cyclin D1/CDK4, 6 or cyclin E/CDK2 complexes (27-29). When these negative cell cycle modulators were examined in the S100P-siRNA transfectants, no significant changes of expression were observed. However, knockdown of S100P elicited down-regulation of cyclin D1 and CDK2 (Fig. 5). These results imply that over-expression of S100P, in HCC cells, might concomitantly activate transcriptional expression of both cyclin D1 and CDK2. This suggests that simultaneous regulation of CDK2 and cyclin D1 by S100P exerts very potent mitogenic stimulation causing uncontrolled cell growth during liver cancer progression.

\section{Discussion}

$\mathrm{S} 100 \mathrm{P}$ is a member of the $\mathrm{S} 100$ family that consists of $\mathrm{Ca}^{2+}$ binding proteins, of the EF-hand type, with at least 20 members $(9,13)$. This protein was first purified from the placenta $(12)$, and has been associated with a large number of diseases by both intracellular and extracellular mechanisms (14). In addition, the S100P protein has been recently considered a potential biomarker of cancer due to its frequent expression in different types of tumor tissues $(15,30)$. However, despite the accumulating evidence supporting aberrant cell cycle regulation and tumorigenic potential of $\mathrm{S} 100 \mathrm{P}$, in many solid tumors, there is no information on its role in human HCC. Much of the experimental data have been obtained by S100Ptransfected cells in vitro and in vivo as well as from DNA micrarray studies (14,15,31-33). We have noted that S100P expression has been shown to gradually increase from dysplasia, precancerous lesions, to HCC based on our previous findings, which suggests large-scale molecular changes from the dysplastic nodule to HCC by expression profiling (34). In addition, these results suggest that dysregulation of S100P might be associated with HCC progression and/or formation. In the present study, the findings showed upregulation of S100P in HCCs; in addition, direct-targeting of RNA interference-mediated protein knockdown of S100P abolished the mitogenic properties of Hep3B cancer cells.

The analysis of endogenous expression of S100P in liver cancer cell lines revealed no detectable mRNA in the THLE-3 cells, which were derived from normal human hepatocytes. However, the cancer cells expressed S100P in both liver cancer cell lines and HCC tissues (Fig. 1). As summarized in Table I, the immunohistochemistry analysis of $25 \mathrm{HCCs}$ showed that 19 out of the 25 tested cases resulted in positive findings $(76 \%)$ in the HCCs; however, 11 cases (44\%) were also positive in the normal tissues. This high rate of positive findings in normal tissues could be partially explained by the presence of background disease such as chronic hepatitis or liver cirrhosis.

With regard to the biological activity of S100P during tumor progression, there are several lines of evidence that indicate that $\mathrm{S} 100 \mathrm{P}$ acts as a proliferative and prosurvival factor in several types of cancer cells including NIH-3T3 cells (35), breast cancer cells (36) and pancreatic cancer cells (14). Our results also showed aberrant expression of S100P in HCCs. To determine the biological role of S100P in HCC, RNA interference-mediated gene silencing was employed. The silencing of endogenous S100P, in the Hep3B cells, resulted in the regression of cell growth (Fig. 3) and increased the basal levels of apoptotic cells (Fig. 4). This result is consistent with previous findings that suggest that S100P might act as an aggressiveness factor in HCC cells by facilitating cell growth and conferring resistance to basal apoptosis.

Accumulating evidence has suggested that S100 proteins have regulatory roles associated with cell cycle transition $(7,37)$. In the G1/S phase transition of the cell cycle, it is wellknown that negative cell cycle regulators such as $\mathrm{p} 21^{\mathrm{WAF} 1 / \mathrm{CIP} 1}$, $\mathrm{p} 15^{\mathrm{INK} 4 \mathrm{~B}}, \mathrm{p} 16^{\mathrm{INK} 4 \mathrm{~A}}$ and $\mathrm{p} 27^{\mathrm{Kip} 1}$ are the key modulators responsible for suppressing cyclin D1/CDK4, 6 or cyclin E/CDK2 complexes (27-29). Thus to identify the underlying mechanism associated with the neoplastic potential of S100P 
aberrant regulation, we investigated the regulatory role of S100P with regard to the key components of the G1/S cell cycle transition. During the regulation of the cell cycle, p $21^{\mathrm{WAF} 1 / \mathrm{CIP} 1}$ inhibits the CDK/cyclin2 complex that induces hypophosphorylation of the $\mathrm{Rb}$ protein. In addition, cyclin $\mathrm{D} 1$ is also involved in regulating the Rb phosphorylation status in association with CDK4/6. As shown in Fig. 5, disruption of S100P simultaneously suppressed the expression of cyclin D1 and CDK2 in the Hep3B cells. This implies that aberrant regulation of S100P might augment two key modulators of the cell cycle exerting synergistic effects on cell cycle transition and may result in uncontrolled cell cycle activity during the development of HCC. Although these findings do not fully explain the mechanisms of S100P that are associated with the cell cycle, it is possible that S100P has a mitogenic role as a cell cycle regulator in Hep3B human hepatocellular carcinoma cells.

According to a recent study, addition of exogenous S100P stimulated cell proliferation and survival via the receptor for activated glycation end products (RAGE). The activation of RAGE is known to phosphorylate Erk and stimulates NF- $\mathrm{KB}$ (35). Erk activation is universally associated with stimulation of cell growth and NF- $\mathrm{BB}$ activation is frequently associated with increased cell survival. Thus, we investigated whether silencing of endogenous S100P by siRNA affected these molecules. However, we observed no significant change in these molecules at either the phosphorylation or protein expression levels (data not shown). Since little is known about the interaction of S100P and Erk 1/2, and the NF- $\mathrm{KB}$ signaling pathway, additional studies are necessary to fully understand the intracellular mechanisms of S100P in liver cancer cells.

In summary, the results of this study showed overexpression of S100P in both cancer cell lines and human HCCs. In addition, disruption of S100P in human HCC cell lines suppressed tumor cell growth by the transcriptional inhibition of CDK2 and cyclin D1. This is the first report that $\mathrm{S} 100 \mathrm{P}$ is dysregulated in human HCC, and to suggest that S100P might contribute to tumor cell growth and survival. The current data suggest that S100P might be associated with the development of the aggressiveness in HCCs.

\section{Acknowledgements}

This work was supported by the Korean Ministry of the Environment via 'The Eco-technopia 21 project' and by the Korean Science \& Engineering Foundation (KOSEF) via the Cell Death Disease Research Center at The Catholic University of Korea.

\section{References}

1. Wong CM and Ng IO: Molecular pathogenesis of hepatocellular carcinoma. Liver Int 28: 160-174, 2008.

2. Befeler AS and Di Bisceglie AM: Hepatocellular carcinoma: diagnosis and treatment. Gastroenterology 122: 1609-1619, 2002.

3. Llovet JM, Bruix J and Gores GJ: Surgical resection versus transplantation for early hepatocellular carcinoma: clues for the best strategy. Hepatology 31: 1019-1021, 2000.

4. Satoh S, Daigo Y, Furukawa Y, et al: AXIN1 mutations in hepatocellular carcinomas, and growth suppression in cancer cells by virus-mediated transfer of AXIN1. Nat Genet 24: 245-250, 2000.
5. De La Coste A, Romagnolo B, Billuart P, et al: Somatic mutations of the beta-catenin gene are frequent in mouse and human hepatocellular carcinomas. Proc Natl Acad Sci USA 95: 8847-8851, 1998.

6. Pang A, Ng IO, Fan ST and Kwong YL: Clinicopathologic significance of genetic alterations in hepatocellular carcinoma. Cancer Genet Cytogenet 146: 8-15, 2003.

7. Zimmer DB, Cornwall EH, Landar A and Song W: The S100 protein family: history, function, and expression. Brain Res Bull 37: 417-429, 1995

8. Schafer BW and Heizmann CW: The S100 family of EF-hand calcium-binding proteins: functions and pathology. Trends Biochem Sci 21: 134-140, 1996.

9. Donato R: S100: a multigenic family of calcium-modulated proteins of the EF-hand type with intracellular and extracellular functional roles. Int J Biochem Cell Biol 33: 637-668, 2001.

10. Heizmann CW, Fritz G and Schafer BW: S100 proteins: structure, functions and pathology. Front Biosci 7: d1356-d1368, 2002.

11. Emoto Y, Kobayashi R, Akatsuka H and Hidaka H: Purification and characterization of a new member of the S-100 protein family from human placenta. Biochem Biophys Res Commun 182: 1246-1253, 1992.

12. Becker T, Gerke V, Kube E and Weber K: S100P, a novel $\mathrm{Ca}(2+)$-binding protein from human placenta. cDNA cloning, recombinant protein expression and $\mathrm{Ca}^{2+}$ binding properties. Eur J Biochem 207: 541-547, 1992.

13. Gribenko A, Lopez MM, Richardson JM, 3rd and Makhatadze GI Cloning, overexpression, purification, and spectroscopic characterization of human S100P. Protein Sci 7: 211-215, 1998.

14. Arumugam T, Simeone DM, Van Golen K and Logsdon CD S100P promotes pancreatic cancer growth, survival, and invasion. Clin Cancer Res 11: 5356-5364, 2005.

15. Gibadulinova A, Barathova M, Kopacek J, et al: Expression of S100P protein correlates with and contributes to the tumorigenic capacity of HeLa cervical carcinoma cells. Oncol Rep 14: 575-582, 2005.

16. Schor AP, Carvalho FM, Kemp C, Silva ID and Russo J: S100P calcium-binding protein expression is associated with high-risk proliferative lesions of the breast. Oncol Rep 15: 3-6, 2006.

17. Birkenkamp-Demtroder K, Olesen SH, Sorensen FB, et al: Differential gene expression in colon cancer of the caecum versus the sigmoid and rectosigmoid. Gut 54: 374-384, 2005.

18. Guerreiro Da Silva ID, Hu YF, Russo IH, et al: S100P calciumbinding protein overexpression is associated with immortalization of human breast epithelial cells in vitro and early stages of breast cancer development in vivo. Int J Oncol 16: 231-240, 2000.

19. Logsdon CD, Simeone DM, Binkley C, et al: Molecular profiling of pancreatic adenocarcinoma and chronic pancreatitis identifies multiple genes differentially regulated in pancreatic cancer. Cancer Res 63: 2649-2657, 2003.

20. Averboukh L, Liang P, Kantoff PW and Pardee AB: Regulation of S100P expression by androgen. Prostate 29: 350-355, 1996.

21. Diederichs S, Bulk E, Steffen B, et al: S100 family members and trypsinogens are predictors of distant metastasis and survival in early-stage non-small cell lung cancer. Cancer Res 64: 5564-5569, 2004.

22. Sato N, Fukushima N, Matsubayashi H and Goggins M: Identification of maspin and S100P as novel hypomethylation targets in pancreatic cancer using global gene expression profiling. Oncogene 23: 1531-1538, 2004.

23. Hammacher A, Thompson EW and Williams ED: Interleukin-6 is a potent inducer of S100P, which is up-regulated in androgenrefractory and metastatic prostate cancer. Int J Biochem Cell Biol 37: 442-450, 2005.

24. Shyu RY, Huang SL and Jiang SY: Retinoic acid increases expression of the calcium-binding protein S100P in human gastric cancer cells. J Biomed Sci 10: 313-319, 2003.

25. Bertram J, Palfner K, Hiddemann W and Kneba M: Elevated expression of S100P, CAPL and MAGE 3 in doxorubicinresistant cell lines: comparison of mRNA differential display reverse transcription-polymerase chain reaction and subtractive suppressive hybridization for the analysis of differential gene expression. Anticancer Drugs 9: 311-317, 1998.

26. Beer DG, Kardia SL, Huang CC, et al: Gene-expression profiles predict survival of patients with lung adenocarcinoma. Nat Med 8: 816-824, 2002.

27. Xiong Y, Hannon GJ, Zhang H, Casso D, Kobayashi R and Beach D: p21 is a universal inhibitor of cyclin kinases. Nature 366: 701-704, 1993. 
28. Grana X and Reddy EP: Cell cycle control in mammalian cells: role of cyclins, cyclin dependent kinases (CDKs), growth suppressor genes and cyclin-dependent kinase inhibitors (CKIs). Oncogene 11: 211-219, 1995.

29. Soto Martinez JL, Cabrera Morales CM, Serrano Ortega S and Lopez-Nevot MA: Mutation and homozygous deletion analyses of genes that control the G1/S transition of the cell cycle in skin melanoma: p53, p21, p16 and p15. Clin Transl Oncol 7: 156-164, 2005.

30. Higgins JP, Kaygusuz G, Wang L, et al: Placental S100 (S100P) and GATA3: markers for transitional epithelium and urothelial carcinoma discovered by complementary DNA microarray. Am J Surg Pathol 31: 673-680, 2007.

31. Hwang MN, Ha TH, Park J, et al: Increased SOCS6 stability with PMA requires its N-terminal region and the Erk pathway via Pkcdelta activation. Biochem Biophys Res Commun 354: 184-189, 2007.

32. Chao A, Wang TH, Lee YS, et al: Molecular characterization of adenocarcinoma and squamous carcinoma of the uterine cervix using microarray analysis of gene expression. Int J Cancer 119 91-98, 2006.
33. Kita H, Hikichi Y, Hikami K, et al: Differential gene expression between flat adenoma and normal mucosa in the colon in a microarray analysis. J Gastroenterol 41: 1053-1063, 2006.

34. Nam SW, Park JY, Ramasamy A, et al: Molecular changes from dysplastic nodule to hepatocellular carcinoma through gene expression profiling. Hepatology 42: 809-818, 2005.

35. Arumugam T, Simeone DM, Schmidt AM and Logsdon CD: S100P stimulates cell proliferation and survival via receptor for activated glycation end products (RAGE). J Biol Chem 279: 5059-5065, 2004

36. Wang G, Platt-Higgins A, Carroll J, et al: Induction of metastasis by $\mathrm{S} 100 \mathrm{P}$ in a rat mammary model and its association with poor survival of breast cancer patients. Cancer Res 66: 1199-1207, 2006.

37. Beissel B, Silva ID, Pesquero JB, Russo J, Schor N and Bellini MH: $\mathrm{S}$-phase reduction in T47D human breast cancer epithelial cells induced by an S100P antisense-retroviral construct. Oncol Rep 17: 611-615, 2007. 